\title{
Gravitational Wave Constraints on DBI Inflation
}

\author{
James E. Lidsey and Ian Huston \\ Astronomy Unit, School of Mathematical Sciences \\ Queen Mary, University of London \\ Mile End Road, London E1 4NS \\ United Kingdom \\ E-mail: J.E.Lidsey@qmul.ac.uk, I.Huston@qmul.ac.uk
}

Submitted to: JCAP

\begin{abstract}
An upper bound on the amplitude of the primordial gravitational wave spectrum generated during ultra-violet DBI inflation is derived. The bound is insensitive to the form of the inflaton potential and the warp factor of the compactified dimensions and can be expressed entirely in terms of observational parameters once the volume of the five-dimensional sub-manifold of the throat has been specified. For standard type IIB compactification schemes, the bound predicts undetectably small tensor perturbations with a tensor-scalar ratio $r<10^{-7}$. This is incompatible with a corresponding lower limit of $r>0.1\left(1-n_{s}\right)$, which applies to any model that generates a red spectral index $n_{s}<1$ and a potentially detectable non-Gaussianity in the curvature perturbation. Possible ways of evading these bounds in more general DBItype scenarios are discussed and a multiple-brane model is investigated as a specific example.
\end{abstract}

Keywords: Inflation, Physics of the early universe. 


\section{Introduction}

The inflationary scenario provides the theoretical framework for the early history of the universe. It has now been successfully tested by observations, including the third year data from the Wilkinson Microwave Anisotropy Probe (WMAP3) [1]. Despite this success, however, the high energy physics that resulted in a phase of accelerated expansion is still not well understood. String/M-theory unifies the fundamental interactions including gravity and it is natural and important to develop inflationary models within string theory and to confront them with cosmological observations.

One class of string-theoretic models that has received considerable attention is Dbrane inflation [2, 3, 4, 15, 6, 7, 8, 9, 10, 11, 12, 13, 14, 15, 16, 17, 18, 19, 20, 21, 22, 23, 24, 25, 26]. (For recent reviews, see, e.g., [27, 28]). Of particular interest is the DBI inflationary scenario [7, 12]. This is based on the compactification of type IIB string theory on a Calabi-Yau (CY) three-fold, where the form-field fluxes generate locally warped regions known as 'throats'. The propagation of a D3-brane in such a region can drive inflation, where the inflaton field is identified with the radial position of the brane along the throat. Since this is an open string mode, the field equation for the inflaton is determined by a Dirac-Born-Infeld (DBI) action.

The purpose of the present work is to explore the observational consequences of DBI inflation. In general, primordial gravitational wave fluctuations and nonGaussian statistics in the curvature perturbation provide two powerful discriminants of inflationary scenarios. The nature of the DBI action is such that the sound speed of fluctuations in the inflaton can be much less than the speed of light. This induces a large and potentially detectable non-Gaussian signal in the density perturbations [7, 12, 29, 30].

The gravitational wave background generated in DBI inflation was recently investigated by Baumann and McAllister (BM) [31]. By exploiting a relationship due originally to Lyth [32], these authors derived a field-theoretic upper limit to the tensor amplitude and concluded that rather stringent conditions would need to be satisfied for these perturbations to be detectable. Moreover, the special case of DBI inflation driven by a quadratic potential is incompatible with the WMAP3 data when this constraint is imposed [33].

Our aim is to derive observational constraints on DBI inflation that are insensitive to the details of the throat geometry and the inflaton potential. In general, there are two realizations of the scenario, which are referred to as the ultra-violet (UV) and infrared (IR) versions, respectively. These are characterized by whether the brane is moving towards or away from the tip of the throat. We focus initially on the UV scenario and derive an upper bound on the gravitational wave amplitude in terms of observable parameters. This limit arises by considering the variation of the inflaton field during the era when observable scales cross the Hubble radius, and we find in general that the tensor-scalar ratio must satisfy $r \lesssim 10^{-7}$. This is below the projected sensitivity of future Cosmic Microwave Background (CMB) polarization experiments [34, 35]. On the 
other hand, the WMAP3 data favours a red perturbation spectrum with $n_{s}<1$ when the tensor modes are negligible and the scalar spectral index is effectively constant [1]. For models which generate such a spectrum, we identify a corresponding lower limit on the tensor modes such that $r \gtrsim 0.1\left(1-n_{s}\right)$. This is incompatible with the upper bound on $r$ when $1-n_{s} \simeq 0.05$, as inferred by the observations.

Hence, a reconciliation between theory and observation requires either a relaxation of the upper limit on $r$ or a blue spectral index $\left(n_{s}>1\right)$. The DBI scenario would need to be generalized in a suitable way for the upper bound on $r$ to be weakened. We identify necessary conditions that a generalized action must satisfy for the BaumannMcAllister constraint to be relaxed. We then show that such conditions can be realized in a recently proposed IR version of DBI inflation driven by multiple coincident branes [36].

The paper is organized as follows. We briefly review DBI inflation in Section 2. In Sections 3 and 4, we derive the respective limits on the amplitude of the tensor perturbations. We determine how the BM bound may be relaxed in generalized DBI models in Section 5 and discuss multi-brane IR scenarios in Section 6. Finally, we conclude in Section 7 . Units are chosen such that $M_{\mathrm{P}} \equiv(8 \pi G)^{-1 / 2}=2.4 \times 10^{18} \mathrm{GeV}$ defines the reduced Planck mass and $c=\hbar=1$.

\section{DBI inflation}

Flux compactification of type IIB string theory to four dimensions results in a warped geometry, where the six-dimensional CY manifold contains one or more throats (see [37, 38] for reviews). The metric inside a throat has the generic form

$$
d s_{10}^{2}=h^{2}(\rho) d s_{4}^{2}+h^{-2}(\rho)\left(d \rho^{2}+\rho^{2} d s_{X_{5}}^{2}\right),
$$

where the warp factor $h(\rho)$ is a function of the radial coordinate $\rho$ along the throat and $X_{5}$ is a Sasaki-Einstein five-manifold. In many cases, the ten-dimensional metric (1) can be approximated locally by the geometry $A d S_{5} \times X_{5}$, where the warp factor is given by $h=\rho / L$ and the radius of curvature of the $A d S_{5}$ space is defined by

$$
L^{4} \equiv \frac{4 \pi^{4} g_{s} N}{\operatorname{Vol}\left(X_{5}\right) m_{s}^{4}},
$$

such that $\operatorname{Vol}\left(X_{5}\right)$ is the dimensionless volume of $X_{5}$ with unit radius, $N$ is the D3 charge of the throat, $g_{s}$ is the string coupling and $m_{s}$ is the string mass scale. In the Klebanov-Strassler (KS) background [39], the throat is a warped deformed conifold and corresponds to a cone over the manifold $X_{5}=T^{1,1}=\mathrm{SU}(2) \otimes \mathrm{SU}(2) / \mathrm{U}(1)$ in the UV limit $(\rho \rightarrow \infty)$. This has a volume $\operatorname{Vol}\left(T^{1,1}\right)=16 \pi^{3} / 27$ and topology $S^{2} \times S^{3}$, where the $S^{2}$ is fibred over the $S^{3}$. The conical singularity at the tip of the throat is smoothed

out by an $S^{3}$ 'cap' due to the wrapping of the fluxes along the cycles of the conifold [39, 40] and the warp factor asymptotes to a constant value in this region.

In general, the low-energy world-volume dynamics of a probe D3-brane in a warped throat is determined by an effective, four-dimensional DBI action. The inflaton field is 
related to the radial position of the brane by $\phi \equiv \sqrt{T_{3}} \rho$, where $T_{3}=m_{s}^{4} /\left[(2 \pi)^{3} g_{s}\right]$ is the brane tension. The action is then given by

$$
\begin{aligned}
& S=\int d^{4} x \sqrt{|g|}\left[\frac{M_{\mathrm{P}}^{2}}{2} R+P(\phi, X)\right] \\
& P(\phi, X)=-T(\phi) \sqrt{1-2 T^{-1}(\phi) X}+T(\phi)-V(\phi),
\end{aligned}
$$

where $R$ is the Ricci curvature scalar, $X \equiv-\frac{1}{2} g^{\mu \nu} \nabla_{\mu} \phi \nabla_{\nu} \phi$ is the kinetic energy of the inflaton, $V(\phi)$ denotes the field's interaction potential and $T(\phi)=T_{3} h^{4}(\phi)$ defines the warped brane tension. We refer to $P(\phi, X)$ as the 'kinetic function' for the inflaton.

We consider a spatially flat and isotropic cosmology sourced by a homogeneous scalar field. In this case, the Friedmann equations for a monotonically varying inflaton can be expressed in the form [7]

$$
\begin{aligned}
& 3 M_{\mathrm{P}}^{2} H^{2}(\phi)=V(\phi)-T(\phi)\left[1-\sqrt{1+4 M_{\mathrm{P}}^{4} T^{-1} H^{\prime 2}}\right] \\
& \dot{\phi}=-\frac{2 M_{\mathrm{P}}^{2} H^{\prime}}{\sqrt{1+4 M_{\mathrm{P}}^{4} T^{-1} H^{\prime 2}}},
\end{aligned}
$$

where $H=H(\phi)$ represents the Hubble parameter as a function of the field and a prime denotes $d / d \phi$.

An important consequence of the non-trivial kinetic structure of the DBI action is that the sound speed of fluctuations in the inflaton differs from unity:

$$
c_{s}=\frac{1}{P_{, X}}=\sqrt{1-2 T^{-1} X}
$$

where a subscripted comma denotes partial differentiation. Furthermore, the condition that the sound speed be real imposes an upper bound on the kinetic energy of the inflaton, $\dot{\phi}^{2}<T(\phi)$, which is independent of the steepness of the potential. The motion of the brane is said to be 'relativistic' when this bound is close to saturation.

We now define the epoch that is directly accessible to cosmological observations as 'observable inflation'. Depending on the reheating temperature, this occurred some 30 to 60 e-foldings before the end of inflation. The inflationary dynamics during this phase can be quantified in terms of three parameters:

$$
\begin{aligned}
& \epsilon \equiv-\frac{\dot{H}}{H^{2}}=\frac{X P_{, X}}{M_{\mathrm{P}}^{2} H^{2}}=\frac{2 M_{\mathrm{P}}^{2}}{\gamma}\left(\frac{H^{\prime}}{H}\right)^{2} \\
& \eta \equiv \frac{2 M_{\mathrm{P}}^{2}}{\gamma} \frac{H^{\prime \prime}}{H} \\
& s \equiv \frac{\dot{c_{s}}}{c_{s} H}=\frac{2 M_{\mathrm{P}}^{2}}{\gamma} \frac{H^{\prime}}{H} \frac{\gamma^{\prime}}{\gamma},
\end{aligned}
$$

where $\gamma \equiv 1 / c_{s}$. We will assume that the 'slow-roll' conditions $\{\epsilon,|\eta|,|s|\} \ll 1$ applied during observable inflation. In this regime, the amplitudes and spectral indices of the two-point functions for the scalar and tensor perturbations are given by [4]

$$
P_{S}^{2}=\frac{1}{8 \pi^{2} M_{\mathrm{P}}^{2}} \frac{H^{2}}{c_{s} \epsilon}=\frac{H^{4}}{4 \pi^{2} \dot{\phi}^{2}}
$$




$$
\begin{aligned}
& P_{T}^{2}=\frac{2}{\pi^{2}} \frac{H^{2}}{M_{\mathrm{P}}^{2}} \\
& 1-n_{s}=4 \epsilon-2 \eta+2 s \\
& n_{t}=-2 \epsilon
\end{aligned}
$$

respectively, where the quantities on the right-hand sides are evaluated when the scale with comoving wavenumber $k=a H \gamma$ crossed the Hubble radius during inflation. The tensor-scalar ratio, $r \equiv P_{T}^{2} / P_{S}^{2}$, is directly related to the tensor spectral index by [41]

$$
r=-8 c_{s} n_{t}=16 c_{s} \epsilon .
$$

Hence, a sound speed different to unity leads to a violation of the standard inflationary consistency equation, which might be detectable in the foreseeable future [42, 43]. Recent observations of the CMB indicate that $P_{S}^{2}=2.5 \times 10^{-9}$ and $r<0.55$ [1]. The best-fit value for a constant spectral index is $n_{s}=0.987_{-0.037}^{+0.019}$ if $r \neq 0$ is assumed as a prior. This is strengthened to $n_{s}=0.948_{-0.018}^{+0.015}$ for a prior of $r=0$ [1].

A further important consequence of a small sound speed is that departures from purely Gaussian statistics may be large [7, 12, 29, 30]. It is conventional to quantify such deviations by expressing the non-Gaussian curvature perturbation $\mathcal{R}$ in the form $\mathcal{R}=\mathcal{R}_{G}+f_{N L}\left(\mathcal{R}_{G}^{2}-\left\langle\mathcal{R}_{G}^{2}\right\rangle\right)$, where $\mathcal{R}_{G}$ represents the Gaussian contribution, the quadratic piece is a convolution and $f_{N L}$ defines the 'non-linearity' parameter [44]. In general, this parameter is a function of the three momenta which form a triangle in Fourier space. However, in the limit where these momenta have equal magnitude, the non-linearity parameter is given to leading-order by [30, 43]

$$
f_{N L}=\frac{1}{3}\left(\frac{1}{c_{s}^{2}}-1\right) .
$$

Currently, WMAP3 constrains this parameter to be $\left|f_{N L}\right| \lesssim 300$ [1, 45].

Finally, Eqs. (17), (11) and (16) may be combined to constrain the warped brane tension during observable inflation:

$$
\frac{T\left(\phi_{*}\right)}{M_{\mathrm{P}}^{4}}=\frac{\pi^{2}}{16} r^{2} P_{S}^{2}\left(1+\frac{1}{3 f_{N L}}\right),
$$

where a subscript ' $*$ ' denotes that the quantity is to be evaluated at that epoch.

In the following, we first consider the UV version of DBI inflation, where the brane moves relativistically towards the tip of the throat. We will assume implicitly that the sound speed is sufficiently small to generate a non-Gaussianity in excess of $f_{N L} \gtrsim 5$, since this is the projected sensitivity of the Planck satellite [46]. Furthermore, we consider an arbitrary warp factor and inflaton potential, subject only to the condition that a sufficiently long phase of quasi-exponential expansion can be maintained to solve the horizon problem of the hot big bang model.

\section{An Upper Bound on the Primordial Gravitational Waves}

Recently, Baumann and McAllister (BM) [31] derived a field-theoretic upper bound on the tensor-scalar ratio by noting that the four-dimensional Planck mass is related 
to the volume of the compactified CY manifold, $V_{6}$, such that $M_{\mathrm{P}}^{2}=V_{6} \kappa_{10}^{-2}$, where $\kappa_{10}^{2} \equiv \frac{1}{2}(2 \pi)^{7} g_{s}^{2} m_{s}^{-8}$. In general, the compactified volume is comprised of bulk and throat contributions, $V_{6}=V_{6, \text { bulk }}+V_{6 \text {,throat }}$, where the latter is given by

$$
V_{6, \text { throat }}=\operatorname{Vol}\left(X_{5}\right) \int_{0}^{\rho_{U V}} d \rho \frac{\rho^{5}}{h^{4}(\rho)},
$$

and $\rho_{U V}$ denotes the radial coordinate at the edge of the throat (defined as the region where $h\left(\rho_{U V}\right)$ is of order unity). If one assumes that the bulk volume is non-negligible relative to that of the throat (i.e. $V_{6 \text {,throat }}<V_{6}$ ), it follows that $M_{\mathrm{P}}^{2}>V_{6 \text {,throat }} \kappa_{10}^{-2}$. For a warped $A d S_{5} \times X_{5}$ geometry, this leads to an upper limit on the total variation of the inflaton field in the throat region:

$$
\frac{\phi_{U V}}{M_{\mathrm{P}}}<\frac{2}{\sqrt{N}}
$$

Condition (19) may be converted into a corresponding limit on the tensor-scalar ratio by noting from the definition (8) that $\dot{\phi}^{2} / M_{\mathrm{P}}^{2}=2 \epsilon H^{2} / P_{, X}$. This implies that the variation of the inflaton field is given by the Lyth bound [32, 31]

$$
\frac{1}{M_{\mathrm{P}}^{2}}\left(\frac{d \phi}{d \mathcal{N}}\right)^{2}=\frac{r}{8},
$$

where $\mathcal{N} \equiv \int d t H$. Since $\phi_{*}<\phi_{U V}$, this results in an upper bound on the observable tensor-scalar ratio [31]:

$$
r_{*}<\frac{32}{N\left(\mathcal{N}_{\text {eff }}\right)^{2}}
$$

where $\mathcal{N}_{\text {eff }} \equiv \int_{0}^{\mathcal{N}_{\text {end }}} d \mathcal{N}\left(r / r_{*}\right)^{1 / 2}$ is a model-dependent parameter that quantifies how $r$ varies during the final stages of inflation.

Typically, one expects $30 \lesssim \mathcal{N}_{\text {eff }} \lesssim 60$, although smaller values may be possible if the slow-roll conditions are violated after observable scales have crossed the horizon. Furthermore, $N \gg 1$ is necessary for backreaction effects to be negligible. Hence, the constraint (21) imposes a strong restriction on DBI inflationary models. On the other hand, the numerical value of $\mathcal{N}_{\text {eff }}$ is uncertain. Our aim here is to focus on the range of values covered by the inflaton field during the observable stages of inflation. This will result in a constraint on the tensor modes that can be expressed in terms of observable parameters.

To proceed, we denote the change in the value of the inflaton field over observable scales by $\Delta \phi_{*}=\sqrt{T_{3}} \Delta \rho_{*}$. Since the brane moves towards the tip of the throat in UV DBI inflation, it follows that $\rho_{*}>\rho_{\text {end }}>0$, which implies that

$$
\rho_{*}>\left|\Delta \rho_{*}\right| \text {. }
$$

This change in the inflaton value will correspond to a fraction of the throat volume, $\left|\Delta V_{6, *}\right|<V_{6, \text { throat }} \lesssim V_{6}$, where equality in the second limit arises if the bulk volume is negligible. Hence, $\left|\Delta \phi_{*}\right|$ is bounded such that

$$
\left(\frac{\Delta \phi}{M_{\mathrm{P}}}\right)_{*}^{2}<\frac{T_{3} \kappa_{10}^{2}\left(\Delta \rho_{*}\right)^{2}}{\left|\Delta V_{6, *}\right|}
$$


The observations of the CMB that directly constrain the primordial tensor perturbations only cover multipole values in the range $2 \leq l \lesssim 100$. This is equivalent to no more than $\Delta \mathcal{N}_{*} \simeq 4$ e-foldings of inflationary expansion and, in general, corresponds to a narrow range of inflaton values. To a first approximation, therefore, the fraction of the throat volume (18) that is accessible to cosmological observation can be estimated to be

$$
\left|\Delta V_{6, *}\right| \simeq \operatorname{Vol}\left(X_{5}\right) \frac{\left|\Delta \rho_{*}\right| \rho_{*}^{5}}{h_{*}^{4}} .
$$

Combining the inequality (22) with Eq. (24) then implies that

$$
\left|\Delta V_{6, *}\right|>\operatorname{Vol}\left(X_{5}\right) \frac{\left(\Delta \rho_{*}\right)^{6}}{h_{*}^{4}},
$$

and substituting the CMB normalization (17) and condition (25) into the bound (23) yields the upper limit

$$
\left(\frac{\Delta \phi}{M_{\mathrm{P}}}\right)_{*}^{6}<\frac{\pi^{3}}{16 \operatorname{Vol}\left(X_{5}\right)} r^{2} P_{S}^{2}\left(1+\frac{1}{3 f_{N L}}\right) .
$$

Hence, employing the Lyth bound in the form $\left(\Delta \phi_{*} / M_{\mathrm{P}}\right)^{2} \simeq r\left(\Delta \mathcal{N}_{*}\right)^{2} / 8$ results in a very general upper limit on the tensor-scalar ratio:

$$
r_{*}<\frac{32 \pi^{3}}{\left(\Delta \mathcal{N}_{*}\right)^{6} \operatorname{Vol}\left(X_{5}\right)} P_{S}^{2}\left(1+\frac{1}{3 f_{N L}}\right) .
$$

Condition (27) is only weakly dependent on the level of non-Gaussianity when $f_{N L}>5$ and we may therefore neglect the factor involving this parameter. Substituting the WMAP3 normalization $P_{S}^{2}=2.5 \times 10^{-9}$ then implies that

$$
r_{*}<\frac{2.5 \times 10^{-6}}{\left(\Delta \mathcal{N}_{*}\right)^{6} \operatorname{Vol}\left(X_{5}\right)} .
$$

Furthermore, the most optimistic estimate for the minimum number of e-foldings that could be probed by observation is $\Delta \mathcal{N}_{*} \simeq 1$, whereas a generic compactification arises when the volume of the Einstein five-manifold is $\operatorname{Vol}\left(X_{5}\right) \simeq \mathcal{O}\left(\pi^{3}\right)$ [39]. This yields a model-independent upper bound on the tensor-scalar ratio for standard UV DBI inflation:

$$
r_{*}<10^{-7}
$$

This is significantly below the sensitivity of future CMB polarization experiments, which will measure $r \gtrsim 10^{-4}$ [34, 35]. If CMB observations are able to span the full range of e-foldings such that $\Delta \mathcal{N}_{*} \simeq 4$, this constraint is strengthened to $r_{*} \lesssim 2 \times 10^{-11}$.

Before concluding this section, we should remark that the estimate (24) was derived under the assumption that the integrand in Eq. (18) is constant. This inevitably introduces errors into the bound (27). However, the two limiting cases of interest in KS-type geometries arise when the warp factor scales either as $h \propto \rho$ or as $h \simeq$ constant [39, 40]. In both cases the integral (18) can be performed analytically. Indeed, if we 
specify $h \propto \rho^{\alpha}$ for some constant $\alpha$, evaluate the integral between $\rho_{*}$ to $\rho_{*}+\Delta \rho_{*}$, and expand to second-order in a Taylor series, we find that

$$
\Delta V_{6, *} \simeq \operatorname{Vol}\left(X_{5}\right) \frac{\rho_{*}^{5}}{h^{4}\left(\rho_{*}\right)}\left(\Delta \rho_{*}\right)\left[1+\frac{(5-4 \alpha)}{2} \frac{\left(\Delta \rho_{*}\right)}{\rho_{*}}\right] .
$$

This implies that the error in Eq. (24) is no greater than about $3\left(\Delta \rho_{*} / \rho_{*}\right)$ if $0 \leq \alpha \leq 1$. More generally, it follows that a similar error will arise for any warp factor $h \propto \rho^{\alpha(\rho)}$, where the function $\alpha(\rho)$ satisfies $0 \leq \alpha(\rho) \leq 1$ over observable scales. We conclude, therefore, that Eq. (24) provides a sufficiently good estimate of the volume element for a generic warp factont

\section{A Lower Bound on the Primordial Gravitational Waves}

The analysis of the previous Section indicates that standard versions of UV DBI inflation generate a tensor spectrum that is unobservably small. Hence, $r=0$ can be assumed as a prior when discussing the WMAP3 data. However, in this case the data excludes a scale-invariant density spectrum at the $3 \sigma$ level when the running in the spectral index, $\alpha_{s} \equiv d n_{s} / d \ln k$, is negligible [1]. Furthermore, a blue spectral index is only marginally consistent with the data when $r \neq 0$ and $\alpha_{s}=0$. (The inferred upper limit is $\left.n_{s}<1.006\right)$. Although the results from WMAP3 do allow for a blue spectrum if there is significant negative running in the spectral index, we will focus in this Section on models that generate a red spectral index $n_{s}<1$, since these seem to be preferred by the current data.

In general, the spectral index may be related to the tensor-scalar ratio. After differentiating Eq. (7) with respect to cosmic time, and employing Eqs. (6) and (13), we find that

$$
1-n_{s}=4 \epsilon+\frac{2 s}{1-\gamma^{2}} \mp \frac{2 M_{\mathrm{P}}^{2}}{\gamma} \frac{T^{\prime}\left|H^{\prime}\right|}{T H},
$$

where the minus (plus) sign corresponds to a brane moving down (up) the warped throat. The second term in Eq. (31) can be converted into observable parameters by defining the 'tilt' of the non-linearity parameter, $n_{N L} \equiv d \ln f_{N L} / d \ln k$ [15]. This implies that $s=-3 f_{N L} n_{N L} /\left[2\left(1+3 f_{N L}\right)\right]$ and substitution of Eqs. (13)-(16) into Eq. (31) then yields

$$
1-n_{s}=\frac{r}{4} \sqrt{1+3 f_{N L}}+\frac{n_{N L}}{1+3 f_{N L}} \mp \sqrt{\frac{r}{8}}\left(\frac{T^{\prime}}{T} M_{\mathrm{P}}\right)_{*} .
$$

In [43], brane inflation near the tip of a KS-type throat was considered, where the warped brane tension asymptotes to a constant value. In this regime, Eq. (32) reduces to the condition $r \simeq 2.3\left(1-n_{s}\right) / \sqrt{f_{N L}}$ when $f_{N L}$ is sufficiently large to be detectable by Planck, i.e., $\left|f_{N L}\right|>5$. It then follows from the WMAP3 best-fit value $n_{s} \simeq 0.987$ and upper limit $\left|f_{N L}\right|<300$ [45] that the gravitational wave amplitude is bounded both

$\ddagger$ As we shall see in the following Section, even an order of magnitude error will make little difference to our final conclusions. 
from above and below such that $0.001 \lesssim r \lesssim 0.01$. These bounds follow from current WMAP3 limits on the spectral index and the non-linearity parameter, but do not take into account the field-theoretic upper bound that must be imposed on the variation of the inflaton field during inflation.

More generally, in UV DBI inflation where the brane moves towards the tip of the throat, it is reasonable to assume that the warp factor decreases monotonically with the radial coordinate over the observable range of inflaton values, i.e., that $d h / d \rho \geq 0$. This condition is satisfied for $A d S_{5} \times X_{5}$ compactifications and KS-type solutions. Consequently, the third term in Eq. (32) will be semi-negative definite, which implies that

$$
\frac{r}{4} \sqrt{1+3 f_{N L}}+\frac{n_{N L}}{1+3 f_{N L}}>1-n_{s} .
$$

Condition (33) is a consistency relation on UV DBI inflation in terms of observable parameters and it may be combined with the upper bound (27) to confront the scenario with observations. Firstly, let us assume that the tensor-scalar ratio is negligible. The WMAP3 data implies that $1-n_{s}>0.037$, and this is only compatible with condition (33) if

$$
n_{N L} \simeq-2 s>3\left(1-n_{s}\right) f_{N L}>0.1 f_{N L} .
$$

However, when $f_{N L} \gg 1$, this would violate the slow-roll conditions that must be satisfied for a consistent derivation of the perturbation spectra (11). For example, the conservative bound $|s|<0.1$ with $1-n_{s} \simeq 0.05$ is violated if $f_{N L}>\mathcal{O}(5)$.

In view of this, let us consider the case where the tensor perturbations are nonnegligible. The magnitude of the second term in condition (33) is suppressed by a factor of $f_{N L}^{3 / 2} \gg 1$ relative to the first. This is expected to be a significant effect in DBI inflation. Consequently, by saturating the WMAP3 limit $\left|f_{N L}\right|<300$ [45], we arrive at a lower bound on the tensor-scalar ratio which applies to any model for which the ratio $\left(n_{N L} / f_{N L}\right)$ is negligible:

$$
r_{*}>\frac{4\left(1-n_{s}\right)}{\sqrt{3 f_{N L}}}>\frac{1-n_{s}}{8} .
$$

This requires $r>0.002$ for the WMAP3 best-fit value $1-n_{s} \simeq 0.013$, which is incompatible with the upper limit (29).

In general, therefore, it is difficult to simultaneously satisfy the bounds on $r$ with the WMAP3 data in standard UV DBI inflation. There is a small observational window where a blue spectrum is consistent with the data, in which case the lower limit (35) does not apply. However, if the tensor modes are negligible, as implied by the inequality (29), the data strongly favours a red spectral index with $n_{s}<0.963$, and this violates the condition (35). A significant detection of a red spectral index requires either a violation of the slow-roll conditions or a sufficiently small value for the volume of $X_{5}$. In particular, combining the limits (28) and (35) results in the condition

$$
\operatorname{Vol}\left(X_{5}\right)<\frac{2 \times 10^{-5}}{\left(1-n_{s}\right)\left(\Delta \mathcal{N}_{*}\right)^{6}},
$$


and we find that $\operatorname{Vol}\left(X_{5}\right) \lesssim 10^{-7}$ for typical values $1-n_{s} \simeq 0.05$ and $\Delta \mathcal{N}_{*} \simeq 4$. This is comparable to the limit on the volume derived for the special case of a quadratic inflaton potential [31]. As noted in [31, 33], condition (36) may be achieved if $X_{5}$ corresponds to a $Y^{p, q}$ space, which has arbitrarily small volume in the limit $q=1$ and $p \rightarrow \infty$ [47. Small volumes could also be realized by orbifolding the $S^{2}$ symmetry of a KS-type throat.

On the other hand, our upper limit (28) on the gravitational waves follows as a consequence of assuming the constraint (22). This could be violated in IR versions of the scenario, where observable scales crossed the Hubble radius when the brane was near the tip of the throat and $\phi \ll M_{\mathrm{P}}$ [13, 15]. Nonetheless, we emphasize that the upper bound (28) on the tensor modes will also apply to any IR DBI model for which $\left|\Delta \phi_{*}\right|<\phi_{*}$. In view of the above discussion, therefore, we will proceed in the following Section to discuss a framework for generalizing the DBI scenario so that the constraints on the tensor modes can be satisfied.

\section{Relaxing the Baumann-McAllister Bound}

In this Section, we take a phenomenological approach and consider a general kinetic function of the form:

$$
P=-f_{1}(\phi) \sqrt{1-f_{2}(\phi) X}-f_{3}(\phi),
$$

where $f_{i}(\phi)$ are unspecified functions of the inflaton field\&. A direct comparison with Eq. (4) indicates that the standard DBI action exhibits two important properties. The first is the condition that $f_{1} f_{2}=2$. This implies that $c_{s} P_{, X}=1$ and greatly simplifies the form of Eq. (20). The second property is that the warp factor uniquely determines

the kinetic structure of the action, i.e., $h^{4} \propto f_{1} \propto f_{2}^{-1}$. In view of this, it is interesting to consider whether the gravitational wave constraints could be weakened by relaxing one or both of these conditions.

Eq. (37) can be transformed into a similar form to that of Eq. (44) through the field redefinition

$$
\varphi \equiv \int d \phi \sqrt{\frac{f_{1} f_{2}}{2}}
$$

This implies that the sound speed of fluctuations in the inflaton is given by

$$
c_{s}=\sqrt{1-f_{2} X}=\frac{f_{1} f_{2}}{2} \frac{1}{P_{, X}},
$$

and the scalar perturbation amplitude by

$$
P_{S}^{2}=\frac{1}{2 \pi^{2}} \frac{H^{4}}{f_{1} f_{2} \dot{\phi}^{2}} .
$$

$\S$ It is assumed implicitly that the functions have a suitable form for generating a successful phase of inflation. 
However, the consistency equation (15) and non-Gaussianity constraint (16) remain unaltered for this more general class of models 43 . It follows, therefore, that the CMB normalization condition (17) generalizes to a constraint on the value of $f_{1}\left(\phi_{*}\right)$ :

$$
\left(\frac{f_{1}}{M_{\mathrm{P}}^{4}}\right)_{*} \simeq \frac{\pi^{2}}{16} r^{2} P_{S}^{2}\left(1+\frac{1}{3 f_{N L}}\right)
$$

Finally, the expression for the scalar spectral index follows most directly by generalizing Eq. (32) via the correspondence (38). It is straightforward to show that

$$
1-n_{s}=\frac{r}{4} \sqrt{1+3 f_{N L}}+\frac{n_{N L}}{1+3 f_{N L}} \mp \sqrt{\frac{r}{4 f_{1} f_{2}}}\left(\frac{f_{1}^{\prime}}{f_{1}} M_{\mathrm{P}}\right)_{*} .
$$

Eq. (38) also allows us to deduce that the infinitesimal variation of the effective field $\varphi$ is given by the Lyth bound, $\left(\Delta \varphi / M_{\mathrm{P}}\right)^{2} \simeq r(\Delta \mathcal{N})^{2} / 8$. This implies that the variation of the inflaton field during inflation is

$$
\int_{0}^{\phi_{*}} \frac{d \phi}{M_{\mathrm{P}}} \sqrt{\frac{f_{1} f_{2}}{2}}=\sqrt{\frac{r_{*}}{8}} \mathcal{N}_{\text {eff }} .
$$

If we now restrict our attention to the observable stage of inflation, and further assume that the variation of $f_{1} f_{2}$ is negligible during that epoch, we find that

$$
\left(\frac{\Delta \phi}{M_{\mathrm{P}}}\right)_{*}^{2} \simeq \frac{\left(\Delta \mathcal{N}_{*}\right)^{2}}{4 f_{1} f_{2}} r_{*}
$$

On the other hand, the BM bound restricts the maximal variation of the scalar field $\phi$ in the throat region. This will be determined by expression (19) for generic warped geometries that are asymptotically $A d S_{5} \times X_{5}$ away from the tip of the throat. Moreover, if observable scales leave the horizon while the brane is inside the throat, the change in the field value must satisfy $\left|\Delta \phi_{*}\right|<\phi_{U V}$. Hence, it follows from Eq. (44) that

$$
r_{*}<\frac{16}{N\left(\Delta \mathcal{N}_{*}\right)^{2}} f_{1} f_{2}
$$

We will refer to condition (45) as the generalized BM bound. Combining expressions (41) and (45) then results in a necessary condition on the value of $f_{2}\left(\phi_{*}\right)$ for the generalized BM bound to be satisfied:

$$
\frac{f_{2}\left(\phi_{*}\right) M_{\mathrm{P}}^{4}}{N}>\frac{\left(\Delta \mathcal{N}_{*}\right)^{2}}{\pi^{2}} \frac{1}{r P_{S}^{2}} .
$$

This condition can also be interpreted as a lower limit on $r$ for a given value of $f_{2}\left(\phi_{*}\right)$.

In UV models, identical arguments that led to the lower limit (35) on the tensorscalar ratio will also apply in this more general context if, as expected, $f_{1}(\phi)$ is a monotonically increasing function. A necessary condition for the lower and upper limits (35) and (45) to be compatible, therefore, is that

$$
f_{1} f_{2}>\frac{N\left(\Delta \mathcal{N}_{*}\right)^{2}\left(1-n_{s}\right)}{4 \sqrt{3 f_{N L}}} .
$$

$\|$ We are being conservative by restricting our discussion to the observable phase of inflation. More generally, if $f_{1} f_{2}$ remains nearly constant over the last $\mathcal{N}$ e-foldings of inflation, we may substitute $\Delta \mathcal{N}_{*} \rightarrow \mathcal{N}_{\text {eff }}$, where $\mathcal{N}_{\text {eff }}$ may be as large as 60. Thus, the generalized bound (45) should be regarded as a necessary (but not sufficient) condition to be satisfied by the tensor modes. 
In IR scenarios, however, the positive sign will apply in the last term of the righthand side of Eq. (42). Hence, assuming $f_{1}^{\prime}>0$ and neglecting the term proportional to $n_{N L} / f_{N L}$ yields an upper limit on the tensor-scalar ratio:

$$
r_{*}<\frac{4\left(1-n_{s}\right)}{\sqrt{3 f_{N L}}} .
$$

Combining conditions (46) and (48) therefore leads to a constraint on $f_{2}\left(\phi_{*}\right)$ for the generalized BM bound to be satisfied in IR inflation:

$$
\frac{f_{2} M_{\mathrm{P}}^{4}}{N}>\frac{\sqrt{3}\left(\Delta \mathcal{N}_{*}\right)^{2}}{4 \pi^{2}} \frac{\sqrt{f_{N L}}}{\left(1-n_{s}\right) P_{S}^{2}} .
$$

To summarize this Section, expression (45) implies that the BM bound (21) could be relaxed if $f_{1} f_{2} \gg 1$ on observable scales. It is therefore important to develop stringinspired models where this condition arises naturally. This will be the focus of the next Section.

\section{IR Inflation and Multiple Branes}

Recently, an interesting version of IR DBI inflation was proposed by Thomas and Ward [36. In this model, the flux annihilation process generates $n$ coincident branes that are initially located at the bottom of a throat region. The dynamics of this configuration is determined by the non-Abelian world-volume theory [48, 49]. This theory exhibits extra stringy degrees of freedom which arise due to the fuzzy nature of the geometry. For the case where a fuzzy two-sphere is embedded in a three-cycle in the $X_{5}$ manifold, the kinetic structure of the action is given in the large $n$ limit by [36]

$$
P=-n T_{3}\left[h^{4}(\phi) W(\phi) \sqrt{1-2 T_{3}^{-1} h^{-4}(\phi) X}-h^{4}(\phi)+V(\phi)\right],
$$

where

$$
W(\phi) \equiv \sqrt{1+C^{-1} h^{-4}(\phi) \phi^{4}}
$$

defines the so-called 'fuzzy' potential, $C=\pi^{2} \hat{C} T_{3}^{2} / m_{s}^{4}$ is a model-dependent constant and $\hat{C} \simeq n^{2}$ is the quadratic Casimir of the $n$-dimensional representation of $\mathrm{SU}(2)$. Comparison with Eq. (37) implies that $f_{1} f_{2}=2 n W$ and $f_{2}=2 /\left(T_{3} h^{4}\right)$. Hence, the new features of this model relative to the standard single-brane scenario are parametrized in terms of the fuzzy potential $W(\phi)$. This configuration is conjectured to be dual to a D5-brane which is wrapped around a two-cycle of the throat [50, 51, 52].

The regime $W \gg 1$ is of interest for relaxing the gravitational wave constraint 4 . The generalized BM bound (46) may now be expressed as a limit on the value of the warp factor $h\left(\phi_{*}\right)$ on CMB scales:

$$
\frac{N T_{3} h_{*}^{4}}{M_{\mathrm{P}}^{4}}<\frac{8 \pi^{2}\left(1-n_{s}\right) P_{S}^{2}}{\sqrt{3 f_{N L}}\left(\Delta \mathcal{N}_{*}\right)^{2}} .
$$

I Note that the case $n \gg 1$ and $W \sim 1$ will not significantly relax the BM bound, since we require $n \ll N$ for backreaction effects to be negligible. 
We now consider whether this limit can be satisfied for reasonable choices of parameters when the warped compactification corresponds to an $A d S_{5}$ or KS throat, respectively. Recall that the warp factor for the $A d S_{5}$ throat is given by $h=\phi /\left(\sqrt{T_{3}} L\right)$. Condition (52) therefore reduces to a constraint on the value of the inflation during observable inflation:

$$
\frac{\phi_{*}^{4}}{M_{\mathrm{P}}^{4}}<\frac{8 \pi^{2}\left(1-n_{s}\right) P_{S}^{2}}{\sqrt{3 f_{N L}}\left(\Delta \mathcal{N}_{*}\right)^{2}} \frac{\lambda}{N},
$$

where $\lambda \equiv \pi N /\left[2 \operatorname{Vol}\left(X_{5}\right)\right]$. However, non-perturbative string effects are expected to become important below a cutoff scale, $\phi_{\text {cut }}=h_{\text {cut }} \lambda^{1 / 4} m_{s}$, where $h_{\text {cut }}$ is the value of the warp factor at that scale. For consistency, therefore, one requires $\phi_{*}>\phi_{\text {cut }}$, which implies an upper limit on the D3-brane charge:

$$
N<\frac{8 \pi^{2}\left(1-n_{s}\right) P_{S}^{2}}{\sqrt{3 f_{N L}}\left(\Delta \mathcal{N}_{*}\right)^{2}}\left(\frac{M_{\mathrm{P}}}{h_{\text {cut }} m_{s}}\right)^{4} .
$$

Assuming the typical values $m_{s} \sim 10^{-2} M_{\mathrm{P}}, \Delta \mathcal{N}_{*} \simeq 4$ and $h_{\text {cut }} \sim 10^{-2}$ implies $N<7 \times 10^{7}\left(1-n_{s}\right) f_{N L}^{-1 / 2}<2 \times 10^{6}$, where the latter inequality follows for $1-n_{s}<0.05$ and $f_{N L}>5$.

For an $A d S_{5}$ throat, the fuzzy potential is a constant and the condition that $W \gg 1$ becomes

$$
\hat{C} \ll \frac{4 \pi^{2} g_{s} N}{\operatorname{Vol}\left(X_{5}\right)} .
$$

Hence, combining inequalities (54) and (55) implies that

$$
\hat{C} \ll \frac{32 \pi^{4}\left(1-n_{s}\right) P_{S}^{2}}{\sqrt{3 f_{N L}}\left(\Delta \mathcal{N}_{*}\right)^{2}} \frac{g_{s}}{\operatorname{Vol}\left(X_{5}\right)}\left(\frac{M_{\mathrm{P}}}{h_{\mathrm{cut}} m_{s}}\right)^{4},
$$

and specifying $g_{s} \sim 10^{-2}$ and $\operatorname{Vol}\left(X_{5}\right) \simeq \pi^{3}$ then yields the limit $\hat{C} \ll 10^{6}\left(1-n_{s}\right) f_{N L}^{-1 / 2}<$ $2 \times 10^{4}$, or equivalently, $n \ll 150$. Furthermore, since $f_{1} f_{2} \simeq$ constant, the inequality (45) may be strengthened by a factor of $\left(\mathcal{N}_{\text {eff }} / \Delta \mathcal{N}_{*}\right)^{2}$ by substituting $\Delta \mathcal{N}_{*} \rightarrow \mathcal{N}_{\text {eff }}$. This ratio could be as high as $(60 / 4)^{2} \simeq 200$, which would rule out this particular model.

Since the branes are initially located at the tip of the throat, another case of interest is the IR limit of the KS geometry, where the warp factor asymptotes to a constant value [53]:

$$
h_{\mathrm{tip}}=\exp \left(-\frac{2 \pi K}{3 M g_{s}}\right),
$$

and $K, M \in \mathbf{Z}^{+}$are the units of flux associated with the NS-NS and R-R three-forms, respectively, such that $N=M K$. In this case, the generalized BM bound (52) becomes

$$
\frac{8 \pi K}{3 M g_{s}}-\ln N>4 \ln \left(\frac{m_{s}}{g_{s}^{1 / 4} M_{\mathrm{P}}}\right)-\ln \left(\frac{64 \pi^{5}\left(1-n_{s}\right) P_{S}^{2}}{\sqrt{3 f_{N L}}\left(\Delta \mathcal{N}_{*}\right)^{2}}\right) .
$$

The radius of the three-sphere at the tip of the KS throat is of the order $\left(g_{s} M\right)^{1 / 2}$ in string units and this must be large (and at the very least should exceed unity) for the supergravity approximation to be reliable. Substituting this requirement into expression 
(58) results in a necessary (but not sufficient) condition on the D3-brane charge for the generalized BM bound to be satisfied:

$$
\frac{1}{N} \exp \left(\frac{8 \pi g_{s} N}{3}\right)>\frac{\sqrt{3 f_{N L}}\left(\Delta \mathcal{N}_{*}\right)^{2}}{64 \pi^{5}\left(1-n_{s}\right) P_{S}^{2}} \frac{1}{g_{s}}\left(\frac{m_{s}}{M_{\mathrm{P}}}\right)^{4} .
$$

Recalling that a necessary condition for the backreaction of the branes to be negligible is $N \gg n \gg 1$ implies that the exponential term in (59) will dominate unless the string coupling constant is extremely small. Hence, for the parameter estimations quoted above, we deduce the lower limit

$$
N-12 \ln N>-6.8+12 \ln \left(\frac{\sqrt{f_{N L}}}{1-n_{s}}\right),
$$

which becomes $N \gtrsim 10^{2}$ for $1-n_{s} \simeq 0.05$ and $f_{N L}>5$.

In general, however, the $K$ and $M$ units of flux are not independent. F-theory compactification on Calabi-Yau four-folds provides a geometric way of parameterizing type IIB string compactifications [54, 55, 56, 57, 53, 58]. Global tadpole cancellation constrains the topology of the four-fold and this restricts the brane and flux configurations. When the KS system is embedded into F-theory, the constraint is given by [53]

$$
\frac{\chi}{24}=n+M K,
$$

where $\chi$ is the Euler characteristic of the four-fold. Hence, $N=M K<\chi / 24$ and together with condition (60), this implies that

$$
\chi>2400,
$$

for $N>10^{2}$. It is known that the Euler number for four-folds corresponding to hypersurfaces in weighted projective spaces can be as high as $\chi \leq 1,820,448$ [58], so there are many compactifications that could in principle satisfy the generalized BM bound. On the other hand, the above limit on the Euler characteristic does allow us to gain some insight into the topology of the extra dimensions, since compactifications which result in a small Euler characteristic would be incompatible with the generalized BM bound.

\section{Conclusion}

In this paper, we have derived an upper limit to the amplitude of the primordial gravitational wave spectrum generated during UV DBI inflation. We considered the maximal inflaton field variation that can occur during the observable stages of inflation and assumed only that the brane was inside the throat during that epoch. The bound (28) is valid for an arbitrary inflaton potential and warp factor (modulo some weak caveats) and can be expressed entirely in terms of observable parameters once the volume of the five-dimensional sub-manifold of the throat has been specified. The inferred upper limit on $r$ is surprisingly strong. We find that the standard UV scenario predicts tensor perturbations that are undetectably small, at a level $r_{*} \lesssim 10^{-7}$. 
The current WMAP3 data favours models that generate a red spectral index $n_{s}<1$ when both the gravitational waves and running in the scalar spectral index are negligible. For UV versions of the scenario, we have identified a corresponding lower limit on $r$ which applies in this region of parameter space, $r_{*} \gtrsim 0.1\left(1-n_{s}\right)$. It is clear that the standard model can not satisfy both the upper and lower bounds on the tensor modes for the observationally favoured value $1-n_{s} \simeq 0.05$.

The generality of our analysis implies that modifying either the inflaton potential or the form of the warp factor is unlikely to resolve this discrepancy. On the other hand, there are a number of possible ways of reconciling theory with observation. In general, either the upper or lower limit on $r$ needs to be relaxed. Weakening the latter would require a violation of the slow-roll conditions or a blue spectral index. A value of $n_{s}>1$ is compatible with WMAP3 if the running of the spectral index is sufficiently negative, but is only marginally consistent if just the tensor modes are non-negligible. The upper limit on $r$ can be weakened by reducing the volume of $X_{5}$ or by generalizing the DBI action. Furthermore, it need not necessarily apply in IR versions of the scenario, although the BM bound will still hold in such cases.

We considered a generalized version of the DBI action and identified a necessary condition on the form of such an action for the BM bound to be relaxed. As a concrete example, we investigated a version of IR inflation that is driven by multiple coincident branes and found that the bounds on the tensor-scalar ratio can indeed be made compatible if the brane charges satisfy appropriate conditions.

The upper bound on $r$ that we derived in section 3 arises because the warp factor in standard DBI models completely specifies the kinetic energy of the inflaton field. Deriving a corresponding bound for the generalized model (37) would be more involved, since the CMB normalization (41) only directly constrains the function $f_{1}(\phi)$ and this may not necessarily depend on the warp factor. Nonetheless, the constraints (23) and (25) can be combined with Eq. (44) to derive a limit on the tensor-scalar ratio in terms of the warp factor and the kinetic function (37) and we find that

$$
r_{*}<\frac{2}{\pi^{2 / 3}\left(\Delta \mathcal{N}_{*}\right)^{2}}\left(\frac{m_{s}}{M_{\mathrm{P}}}\right)^{4 / 3} \frac{h_{*}^{4 / 3}\left(f_{1} f_{2}\right)_{*}}{g_{s} \operatorname{Vol}\left(X_{5}\right)} .
$$

For a specific model where the warp factor and the functions $f_{i}(\phi)$ are determined by particle physics considerations, condition (63) may be interpreted as a bound that relates the tensor modes directly to the value of the inflaton field during observable inflation. This constraint provides a consistency check that any given model must satisfy irrespective of the form of the inflaton potential.

In conclusion, therefore, primordial gravitational wave constraints combined with cosmological observations of the density perturbation spectrum act as a powerful discriminant of DBI inflationary models. They also serve as an important observational guide for identifying viable generalizations of the scenario. 


\section{Acknowledgments}

IH is supported jointly by a Queen Mary studentship and the Science and Technology Facilities Council (STFC). We thank S. Thomas and J. Ward for helpful discussions.

\section{References}

[1] Spergel D N et al. (WMAP), Wilkinson Microwave Anisotropy Probe (WMAP) three year results: Implications for cosmology, 2006 astro-ph/0603449

[2] Dvali G R and Tye S H H, Brane inflation, 1999 Phys. Lett. B450 72-82 hep-ph/9812483.

[3] Dvali G R, Shafi Q and Solganik S, D-brane inflation, 2001 hep-th/0105203

[4] Burgess C P, Majumdar M, Nolte D, Quevedo F, Rajesh G and Zhang R J, The inflationary brane-antibrane universe, 2001 JHEP 07047 hep-th/0105204

[5] Kachru S, Kallosh R, Linde A and Trivedi S P, De Sitter vacua in string theory, 2003 Phys. Rev. D68 046005 hep-th/0301240

[6] Kachru S, Kallosh R, Linde A, Maldacena J, McAllister L and Trivedi S P, Towards inflation in string theory, 2003 JCAP 0310013 hep-th/0308055

[7] Silverstein E and Tong D, Scalar speed limits and cosmology: Acceleration from D-cceleration, 2004 Phys. Rev. D70 103505 hep-th/0310221

[8] Shandera S, Shlaer B, Stoica H and Tye S H H, Inter-brane interactions in compact spaces and brane inflation, 2004 JCAP 0402013 hep-th/0311207

[9] Firouzjahi H and Tye S H H, Closer towards inflation in string theory, 2004 Phys. Lett. B584 147-154 hep-th/0312020

[10] Burgess C P, Cline J M, Stoica H and Quevedo F, Inflation in realistic D-brane models, 2004 JHEP 09033 hep-th/0403119]

[11] Iizuka N and Trivedi S P, An inflationary model in string theory, 2004 Phys. Rev. D70 043519 hep-th/0403203

[12] Alishahiha M, Silverstein E and Tong D, DBI in the sky, 2004 Phys. Rev. D70 123505 hep-th/0404084

[13] Chen X, Multi-throat brane inflation, 2005 Phys. Rev. D71 063506 hep-th/0408084

[14] Chen X, Inflation from warped space, 2005 JHEP 08045 hep-th/0501184

[15] Chen X, Running non-Gaussianities in DBI inflation, 2005 Phys. Rev. D72 123518 astro-ph/0507053

[16] Cline J M and Stoica H, Multibrane inflation and dynamical flattening of the inflaton potential, 2005 Phys. Rev. D72 126004 hep-th/0508029

[17] Frey A R, Mazumdar A and Myers R, Stringy effects during inflation and reheating, 2006 Phys. Rev. D73 026003 hep-th/0508139

[18] Chialva D, Shiu G and Underwood B, Warped reheating in multi-throat brane inflation, 2006 JHEP 01014 hep-th/0508229

[19] Brodie J H and Easson D A, Brane inflation and reheating, 2003 JCAP 0312004 hep-th/0301138

[20] Vikman A, Inflation with large gravitational waves, 2006 astro-ph/0606033

[21] Mukhanov V F and Vikman A, Enhancing the tensor-to-scalar ratio in simple inflation, 2006 JCAP 0602004 astro-ph/0512066

[22] Kallosh R and Linde A, Testing String Theory with CMB, 2007 JCAP 0704017 arXiv:0704.0647[hep-th]

[23] Shandera S E and Tye S H H, Observing brane inflation, 2006 JCAP 0605007 hep-th/0601099

[24] Chen X and Tye S H H, Heating in brane inflation and hidden dark matter, 2006 JCAP 0606011 hep-th/0602136 
[25] Kecskemeti S, Maiden J, Shiu G and Underwood B, DBI inflation in the tip region of a warped throat, 2006 JHEP 09076 hep-th/0605189

[26] Shiu G and Underwood B, Observing the geometry of warped compactification via cosmic inflation, 2007 Phys. Rev. Lett. 98051301 hep-th/0610151

[27] Tye S H, Brane inflation: String theory viewed from the cosmos, 2006 hep-th/0610221

[28] Cline J M, String cosmology, 2006 hep-th/0612129

[29] Seery D and Lidsey J E, Primordial non-gaussianities in single field inflation, 2005 JCAP 0506 003 astro-ph/0503692

[30] Chen X, Huang M-x, Kachru S and Shiu G, Observational signatures and non-Gaussianities of general single field inflation, 2007 JCAP 0701002 hep-th/0605045

[31] Baumann D and McAllister L, A microscopic limit on gravitational waves from D-brane inflation, 2006 hep-th/0610285

[32] Lyth D H, What would we learn by detecting a gravitational wave signal in the cosmic microwave background anisotropy?, 1997 Phys. Rev. Lett. 78 1861-1863 hep-ph/9606387

[33] Bean R, Shandera S E, Henry Tye S H and Xu J, Comparing brane inflation to WMAP, 2007 JCAP 0705004 hep-th/0702107

[34] Taylor A C et al., Clover: A new instrument for measuring the B-mode polarization of the CMB, 2004 astro-ph/0407148

[35] Verde L, Peiris $\mathrm{H}$ and Jimenez R, Optimizing CMB polarization experiments to constrain inflationary physics, 2006 JCAP 0601019 astro-ph/0506036

[36] Thomas S and Ward J, IR inflation from multiple branes, 2007 hep-th/0702229

[37] Grana M, Flux compactifications in string theory: A comprehensive review, 2006 Phys. Rept. 423 91-158 hep-th/0509003

[38] Douglas M R and Kachru S, Flux compactification, 2006 hep-th/0610102

[39] Klebanov I R and Strassler M J, Supergravity and a confining gauge theory: Duality cascades and $\chi S B$-resolution of naked singularities, 2000 JHEP 08052 hep-th/0007191

[40] Klebanov I R and Tseytlin A A, Gravity duals of supersymmetric $S U(N) \times S U(N+M)$ gauge theories, 2000 Nucl. Phys. B578 123-138 hep-th/0002159

[41] Garriga J and Mukhanov V F, Perturbations in k-inflation, 1999 Phys. Lett. B458 219-225 hep-th/9904176

[42] Lidsey J E and Seery D, An observational test of holographic inflation, 2006 Phys. Rev. D73 023516 astro-ph/0511160

[43] Lidsey J E and Seery D, Primordial non-Gaussianity and gravitational waves: Observational tests of brane inflation in string theory, 2007 Phys. Rev. D75 043505 astro-ph/0610398

[44] Maldacena J M, Non-Gaussian features of primordial fluctuations in single field inflationary models, 2003 JHEP 05013 astro-ph/0210603

[45] Creminelli P, Senatore L, Zaldarriaga $\mathrm{M}$ and Tegmark M, Limits on $f_{N L}$ parameters from WMAP 3yr data, 2007 JCAP 0703005 astro-ph/0610600

[46] Planck project, URL http://www.rssd.esa.int/index.php?project=Planck

[47] Gauntlett J P, Martelli D, Sparks J and Waldram D, Sasaki-Einstein metrics on S(2) x S(3), 2004 Adv. Theor. Math. Phys. 8 711-734 hep-th/0403002

[48] Myers R C, Dielectric-branes, 1999 JHEP 9912022 hep-th/9910053

[49] Myers R C, Nonabelian phenomena on D-branes, 2003 Class. Quantum Grav. 20 S347 hep-th/0303072

[50] Thomas S and Ward J, Non-Abelian $(p, q)$ strings in the warped deformed conifold, 2006 JHEP 12 057 hep-th/0605099

[51] Firouzjahi H, Dielectric (p,q) strings in a throat, 2006 JHEP 12031 hep-th/0610130

[52] Bachas C, Douglas M R and Schweigert C, Flux stabilization of D-branes, 2000 JHEP 05048 hep-th/0003037

[53] Giddings S B, Kachru S and Polchinski J, Hierarchies from fluxes in string compactifications, 2002 Phys. Rev. D66 106006 hep-th/0105097 
[54] Witten E, Phase transitions in M-theory and F-theory, 1996 Nucl. Phys. B471 195 hep-th/9603150

[55] Sethi S, Vafa C and Witten E, Constraints on low-dimensional string compactifications, 1996 Nucl. Phys. B480 213 hep-th/9606122

[56] Gukov S, Vafa C and Witten E, CFT's from Calabi-Yau four-folds, 2000 Nucl. Phys. B584 69 hep-th/9906070

[57] Dasgupta K, Rajesh G and Sethi S, M-theory, orientifolds and G-flux, 1999 JHEP 9908023 hep-th/9908088

[58] Klemm A, Lian B, Roan S S and Yau S T, Calabi-Yau fourfolds for $M$ - and F-theory compactifications, 1998 Nucl. Phys. B518 515 hep-th/9701023 